This work was made possible by a grant-in-aid from the National Research Council of Canada. Margaret A. Graham

Department of Microscopic Anatomy,

University of Western Ontario,

London, Ceanada.

Oct. 6.

${ }^{1}$ Barr, M. L., and Bertram, E. G., Nature, 163, 676 (1949). Barr, Bertram, L. F., and Lindsay, H. A., Anat. Rec., 107, 283 (1950). Barr, M. L., Exp. Cell Res., 2, 288 (1951).

- Graham, M. A., and Barr, M. L., Anat. Rec., 112, 709 (1952).

'Moore, K. L., and Barr, M. L., J. Comp. Neurol., 98, 213 (1953).

\section{Influence of Position of Truss on Pleiocotyly in Tomato}

Lewis $^{1}$ has shown that position of truss affects the manifestation of rogues in tomato. Darlington and Mather ${ }^{2}$ believe this character is plasmagenically inherited, like other forms of rogues in inbreeding species. An investigation was therefore made to determine whether truss position and plasmagenes also influence pleiocotyly, that is, the variation in cotyledon number, in tomato (Lycopersicon esculentum).

Eight plants of Clucas-99 variety were used, and fruits were harvested from individual trusses. Seeds from each were sown. Fig. 1 gives the mean amount of pleiocotyly occurring in offspring from all plants plotted against truss position. This shows that there is a decrease in pleiocotyly from the first to the third truss, with a rise at the fourth. This is converse to that for roguing and truss position found by Lewis ${ }^{1}$. An analysis of variance shows that $P=0.01$ for 'trusses' and $P>0.20$ for 'plants'. Hence, truss position is important in effecting manifestation of pleiocotyly in the offspring; but plants do not differ significantly from one another in their effects, which is not unexpected in an inbred.

As the data strongly indicate that truss position influences pleiocotyly production, and because rogues in tomatoes are possibly plasmagenically controlled, a test was made to see whether rogues and pleiocotyly are associated. One might expect that rogues would produce more pleiocotyls than normal plants if pleiocotyly were influenced by eytoplasmic factors. Table 1 gives data from Clucas-99. A $\chi^{2}$ analysis for independence gives $\chi^{2}=1.53$ and $P>0.20$. Hence roguing and pleiocotyly are independent of each other; however, pleiocotyls produce twice as many rogues. This provides further evidence that cytoplasmic effects are not primarily responsible for the production of pleiocotyls, but might play some part in their activities.

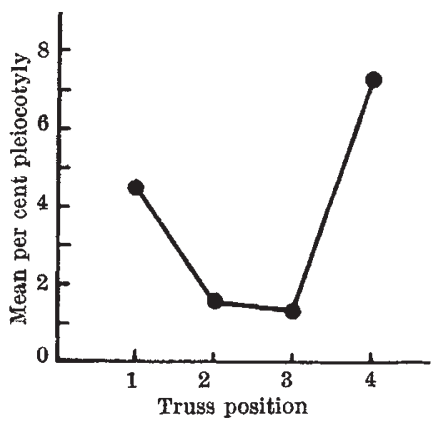

Fig. 1. Per cent pleiocotyly frequency in Clucas-99 tomato variety plotted against position of truss (lowest is 1 , highest is 4) Each spot represents the mean of eight plants
Table 1. Distribution of Pleiocotyly in Normal and Rogui

\begin{tabular}{|l|c|c|}
\multicolumn{2}{c|}{ CLUCAS-99 ToMATo } \\
\hline Type of pleiocotyly & Normal seedlings & Rogue seedlings \\
\hline Monocot. & 1 & 0 \\
Dicot. & 27 & 7 \\
Dicot.-abnormal & 2 & 1 \\
Pseudo-tricot. & 4 & 7 \\
Tricot. & 9 & 1 \\
Pseudo-tetracot. & 2 & 0 \\
Tetracot. & 1 & 17 \\
\hline \multicolumn{1}{|c|}{ Total } & 46 & \\
\hline
\end{tabular}

Table 2. Pheiocotyly IN StonfR's Exhibition TOMATo

\begin{tabular}{|l|c|c|c|}
\hline \multicolumn{1}{|c|}{ Cross } & $\begin{array}{c}\text { Dicot. } \\
\text { (per cent) }\end{array}$ & $\begin{array}{c}\text { Pleiocot. } \\
\text { (per cent) }\end{array}$ & No. of rogues \\
\hline Normal selfed & 100 & 0 & 0 \\
Rogue $\times$ normal & 96 & 4 & 0 \\
Normal $\times$ rogue & 93 & 7 & 1 pleiocot. \\
\hline
\end{tabular}

Data were also available for Stoner's Exhibition variety. Dr. D. Lewis (unpublished) has found the distribution of pleiocotyly as given in Table 2. Here again pleiocotyls were not rogues; but pleiocotyls increased in proportion following reciprocal crosses between rogues and normal. This suggests that dicotyly is a condition partly resulting from balance between chromosomes and cytoplasm. When one or other is disturbed, the threshold for dicotyly is broken down and pleiocotyly results.

Gordon Haskell

John Innes Horticultural Institution, Hertford.

Nov. 2.

${ }^{1}$ Lewis, D., Ann. Rep. John Innes Hort. Inst., 1949, 11 (1950). " Darlington, C. D., and Mather, K." "The Elements of Genetics"

\section{Some Polyenes from Brassica rutabaga}

RECENT studies on fruit and vegetable processing have shown some natural constituents of the material to be very sensitive to treatments, particularly heat. In the course of dehydration experiments with roots of Brassica rutabaga, the pigmentation of the tissues has been examined in detail.

Extraction of the pigment was carried out in the dark by means of a low-temperature technique ${ }^{1}$, and subsequent chromatographic analysis of the solution on an alumina column yielded several polyenes adsorbed less strongly than all-trans-lycopene. Their absorption spectra after repeated chromatography showed single absorption maxima in the region $420-450 \mathrm{~m} \mu$ with no fine structure, and all except No. 5 failed to agree with known maxima either for naturally occurring poly-cis-compounds ${ }^{2,3}$ or for poly-cis-compounds derived from the isomerization of prolycopene $e^{4}$. These differences cannot easily be explained by variations in solvent and spectrophotometer. In each case it was possible to obtain all-trans-lycopene from the solution of chromatographically pure pigment by isomerization with iodine ${ }^{5}$. These pigments are, therefore, hitherto uncharacterized poly-cis-lycopenes. Pigment No. 5 showed a close resemblance to poly-cis-lycopene IV isolated from pyracantha berries by Zechmeister ${ }^{2}$.

We believe that these pigments should have a more distinctive numbering than that referring to their position on an adsorption column, and make the following proposal, namely, that the figure for 